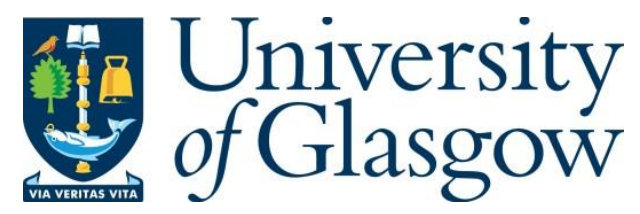

Li, H., Liang, X., Shrestha, A., Liu, Y., Heidari, H., Le Kernec, J. and Fioranelli, F. (2019) Hierarchical sensor fusion for micro-gestures recognition with pressure sensor array and radar. IEEE Journal of Electromagnetics, RF and Microwaves in Medicine and Biology, (doi:10.1109/JERM.2019.2949456).

There may be differences between this version and the published version. You are advised to consult the publisher's version if you wish to cite from it.

http://eprints.gla.ac.uk/187528/

Deposited on: 31 May 2019

Enlighten - Research publications by members of the University of Glasgow http://eprints.gla.ac.uk 


\section{Hierarchical Sensor Fusion for Micro-Gesture Recognition with Pressure Sensor Array and Radar}

Haobo. Li, Xiangpeng. Liang, Aman. Shrestha, Student Member, IEEE, Yuchi. Liu, Hadi. Heidari, Julien. Le Kernec and Francesco. Fioranelli, Senior Member, IEEE

Sensor Data Processing Classifier
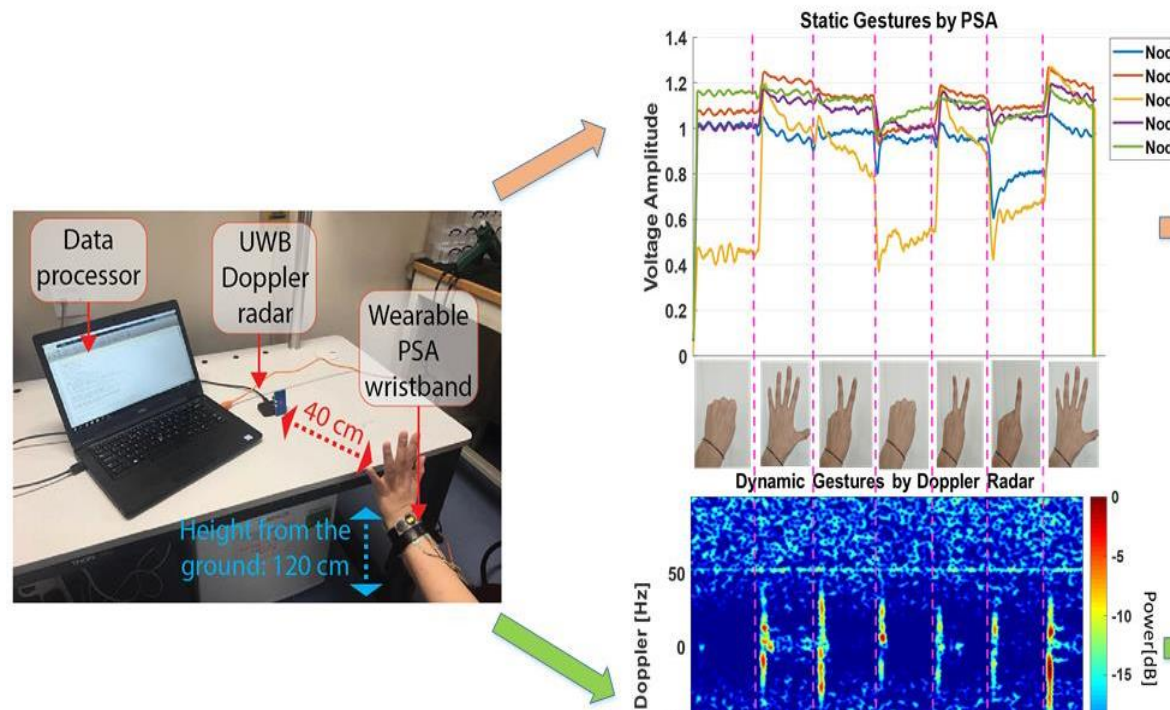

- Node5

Soft Fusion

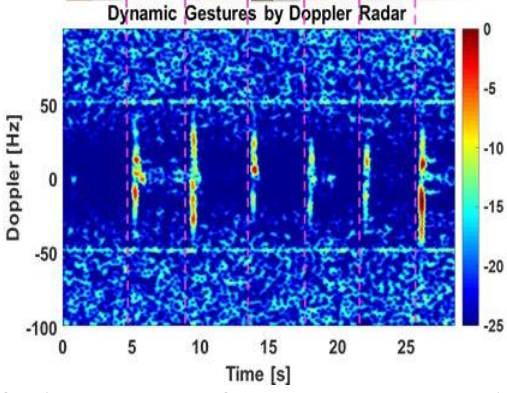
Class Score $\sum W(x)$

The schematic of the hierarchical sensor fusion system for gesture recognition.

\section{Take-Home Messages}

- We proposed a hierarchical model to utilize radar as an 'Enhancer' to complement with the PSA (Pressure Sensor Array) in improving the static gestures recognition rates, on the contrary, in the dynamic gesture case scenario the PSA acts as an 'Enhancer' to boost the radar performance.

- Sequential forward selection (SFS) significantly reduces the computational intensity in terms of less features and improves the classification performance.

- For the second-stage of the hierarchical model, soft and hard fusion methods are implied respectively to promote the classification accuracy and eliminate the false alarms. Different weights of the 'Enhancer' output are verified and compared in terms of the accuracy in the soft fusion process.

- Soft fusion improves the accuracy by $16.7 \%$ and $11.1 \%$ with respect to static and dynamic gesture identification, whereas hard fusion reduces the accuracy variance across all the participants and produces a subsequent improvement about $5.5 \%$ in the dynamic gestures.

- Future work involves more gestures and more participants with neural network-based algorithm and additional sensors configurations and fusion approaches. 


\title{
Hierarchical Sensor Fusion for Micro-Gestures Recognition with Pressure Sensor Array and Radar
}

\author{
Haobo. Li, Xiangpeng. Liang, Aman. Shrestha, Student Member, IEEE, Yuchi. Liu, Hadi. \\ Heidari, Julien. Le Kernec and Francesco. Fioranelli, Senior Member, IEEE
}

\begin{abstract}
This paper presents a hierarchical sensor fusion approach for human micro-gesture recognition by combining an Ultra Wide Band (UWB) Doppler radar and wearable pressure sensors. First, the wrist-worn pressure sensor array (PSA) and Doppler radar are used to respectively identify static and dynamic gestures through a Quadratic-kernel SVM (Support Vector Machine) classifier. Then, a robust wrapper method is applied on the features from both sensors to search the optimal combination. Subsequently, two hierarchical approaches where one sensor acts as "enhancer" of the other are explored. In the first case, scores from Doppler radar related to the confidence level of its classifier and the prediction label corresponding to the posterior probabilities are utilized to maximize the static hand gestures classification performance by hierarchical combination with PSA data. In the second case, the PSA acts as an 'Enhancer' for radar to improve the dynamic gesture recognition. In this regard, different weights of the 'Enhancer' sensor in the fusion process have been evaluated and compared in terms of classification accuracy. A realistic cross-validation method is chosen to test one unknown participant with the model trained by data from others, demonstrating that this hierarchical fusion approach for static and dynamic gestures yields approximately $15 \%$ improvement in classification accuracy in the best cases.
\end{abstract}

Keywords - multimodal sensing, gesture classification, UWB Doppler radar, machine learning

\section{INTRODUCTION ${ }^{1}$}

Micro-gesture recognition [1], [2] has gained significant interest in the context of industrial precision control [3], human-machine interaction, automotive driving assistance [4] and remote medical aid including emergency examination and surgery [5]. Compared with the past decades, more sensing technologies are available in the commercial market due to the rapid development of wireless network and miniaturized fabrication.

The most mature sensing approach, notably wearable devices including Inertial Measurement Unit (IMU) [6], magnetic Hall sensor [7], pressure sensor and barometer [3], [8], [9] require users to carry the sensor during their daily lives. They may dislike the feeling of being constrained or simply forget to wear the device when needed. In the past few years, contactless sensors involving RF-based devices [10] and video camera-based system [11] have been proposed to achieve less intrusive and more comfortable user experience than wearable devices to monitor human movements, including gestures. Compared with cameras, RF-based and radar devices can be perceived

This paper is an extended paper from the one presented in the IEEE Sensors Conference, held in New Delhi, India on 30/10 - 01/11 2018.

The authors acknowledge support of EPSRC grant EP/R041679/1 and Doctoral Training Award supporting A. Shrestha in his $\mathrm{PhD}$ at the School of Engineering, University of Glasgow.

All authors are with the School of Engineering, University of Glasgow, G12 8QQ, Glasgow, UK (e-mail: francesco.fioranelli@glasgow.ac.uk; Julien.lekernec@glasgow.ac.uk) as less intrusive, especially in private home environments, and are less sensitive to environmental light conditions [1].

Micro-gestures are comprised of sequences of static parts (when hand and fingers are stationary) and dynamic transitions between static states [1]. Radar can well detect the transitions due to the Doppler Effect caused by the movement of hand and fingers, whereas the static targets are more challenging and can be ignored as background clutter (unless the radar is very sensitive and with very fine range and angular resolution). On the contrary, wearables such as the PSA (Pressure Sensor Array) can provide meaningful readings for the static and dynamic gestures simultaneously, converting the tendons pressure into voltages. The different information from these sensors can be mutually complementary, improving the overall classification performance.

In this paper, we expand our work on the hierarchical classification of human activities and fall detection [12] by exploring hierarchy in the multimodal sensing framework rather than in the division of classes in sub-groups. Instead of dividing the gestures into different sub-groups and applying further classification algorithms, the proposed hierarchical fusion combines the outputs of individual sensor accounting for their diverse information and exploring the effect of different weights of one against another. Regarding to the sensor fusion algorithm, soft fusion [13] using the classifiers' confidence level, and hard fusion [14] with respect to the prediction labels are tested and compared, considering different weights of the 'Enhancer' sensor's output in the soft fusion. 


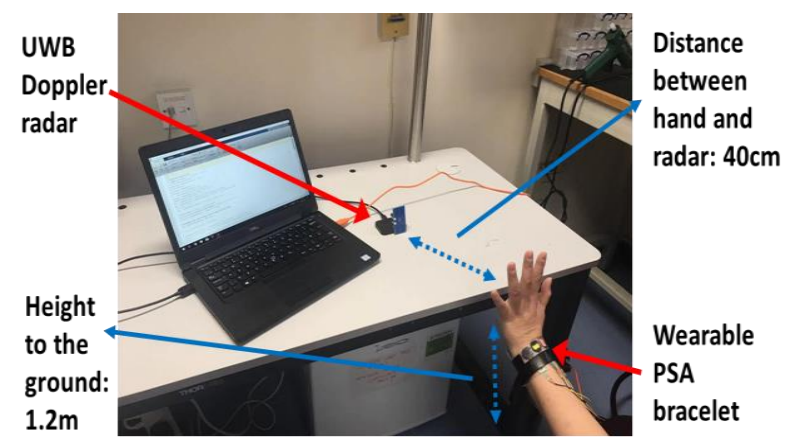

Fig. 1 Experimental setup (on the participant wrist: wearable pressure sensor array bracelet, blue chip on the box: UWB pulse-Doppler radar).

This paper is organized as follows: Section II describes the experimental setup and data collection; Section III discusses the feature selection and proposed hierarchical architecture; the conclusion is summarized in Section IV and some potential further work are indicated.

\section{EXPERIMENTAL SETUP}

The data were collected at the University of Glasgow meLAB laboratory (shown in Fig. 1) with ten male participants, aged from 21 to 36 . In the experiment, the participants were asked to perform 4 different static gestures (number $0,1,2$, and 5 with their left hand), and the transitions between pairs of these static gestures. The subjects were asked to keep the same static gesture for approximately $4 \mathrm{~s}$ then change to the next static gesture. The dataset includes seven instances of static gestures and six transitions. Therefore, the length of the data frame for single participant is approximately $28 \mathrm{~s}$.

As shown in Fig. 1 an off-the-shelf UWB Doppler radar and one PSA (Pressure Sensor Array) wristband were utilized to acquire the data and transmit it to the connected laptops simultaneously. The PSA wristband was placed on the left hand of the participants to measure the pressure amplitudes at five different positions on the human wrist (for tendons of the five fingers), whereas the radar captured range and velocity information of the hand's movement during the gesture transitions.

For the PSA wristband, five Force Sensitive Resistor (FSR402) nodes and one Arduino DUE were utilized to construct the sensor array and then convert the pressure to the voltage reading accordingly. The data were collected through LABVIEW interface with a sampling rate of $50 \mathrm{~Hz}$. The radar was placed on a plastic table at approximately $1.2 \mathrm{~m}$ height to the ground and it pointed to the middle of the hand at a distance of approximately $40 \mathrm{~cm}$. The UWB Pulse-Doppler radar (Xethru X4M300) operated at a center frequency of the transmitter equal to $7.29 \mathrm{GHz}$, with approximate $1.5 \mathrm{GHz}$ useful bandwidth at $-10 \mathrm{~dB}$. The transmitted PRF (Pulse Repetition Frequency) was equal to $200 \mathrm{~Hz}$. The radar signal is digitized as a matrix of complex numbers with amplitude and phase, for further processing using MATLAB.

The voltage readings of PSA wristband from one participant and relative radar Doppler signature are

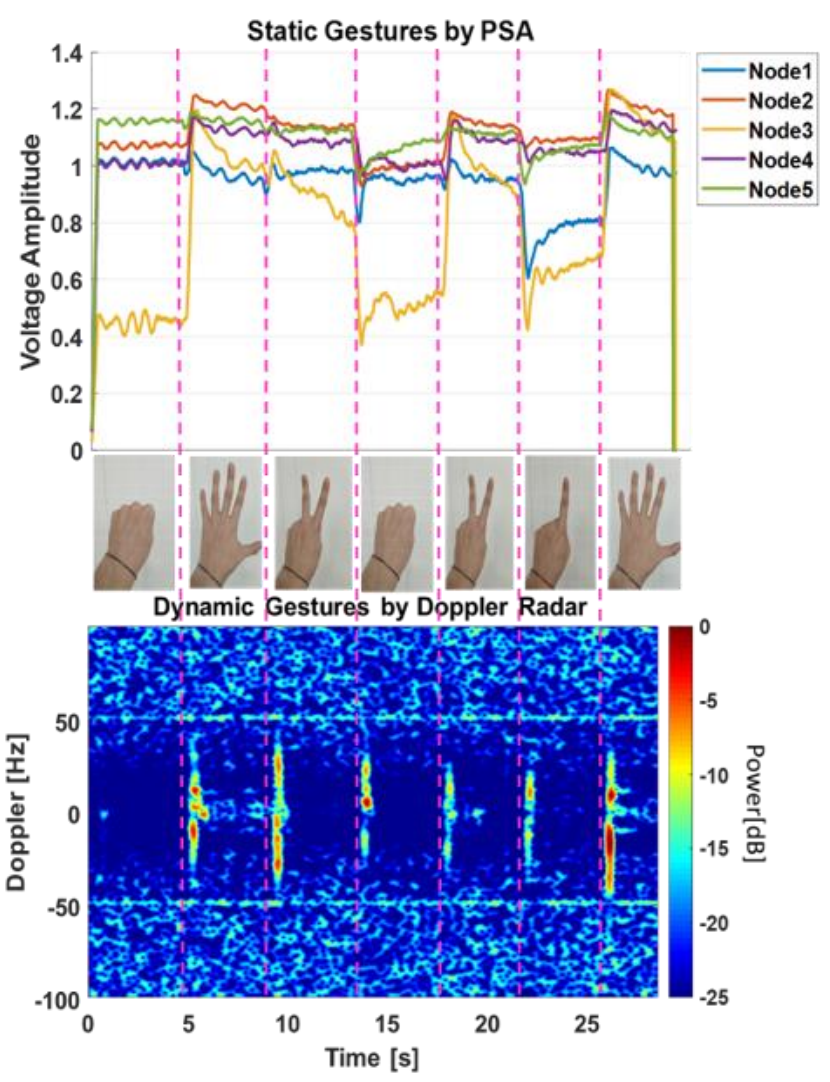

Fig. 2 Static gestures data (top), radar Doppler signature (bottom).

illustrated in Fig. 2 as an example. The PSA produces a flat response when the hand is static translating the pressure from tendons into voltages. The radar data are based on the Doppler-effect, in particular micro-Doppler effect [15], and are more sensitive to moving targets, in our case to the transitions between each static gesture.

The dimension of the dataset is $6 * 7 * 6$ and $6 * 6 * 6$ (number of participants*number of observations in $28 \mathrm{~s}$ *degrees of freedom) for static and dynamic gesture respectively. The degrees of freedom contains five pressure sensor nodes and one UWB radar.

\section{ClassificAtion Results}

\section{A. Feature extraction and selection}

Due to the difference in wrist size, tendons' strength, and sensor node positions for each participant, the numerical data from PSA are quite different in amplitude from one participant to another. To process them on the same scale, the raw data are standardized by subtracting the mean value and dividing the standard deviation. Instead of using raw data to feed the SVM classifier, 29 statistical features [16], [17] listed in Table I are chosen as more compact and representative information to characterize the data. These include: the two dimensional mean value, maximum, minimum and range of all sensor nodes data for each static gestures (4 features in total), as well as the mean and standard deviation of the correlation function to represent the relationship between pairs of sensor nodes ( 20 features in total as there are 5 sensor nodes, 10 different combos). For increasing the robustness of the dynamic gesture 
classification, 5 more features, 1 for each resistor of the PSA, have been utilized. These are the difference between the mean of first 50 data points and the mean of last 50 data points in order to estimate the pressure difference between the previous and next static gestures.

TABLE I LIST OF STATISTICAL FEATURES FROM PSA
\begin{tabular}{|l|c|}
\hline PSA Features & No. \\
\hline 2-d Mean of the voltage amplitude & 1 \\
\hline Max of the voltage amplitude & 1 \\
\hline Min of the voltage amplitude & 1 \\
\hline Range of the voltage amplitude & 10 \\
\hline $\begin{array}{l}\text { Mean of the cross-correlation between data from Node } \\
x \text { and Node y }\end{array}$ & 10 \\
\hline $\begin{array}{l}\text { Standard deviation of the cross-correlation between } \\
\text { data from Node } x \text { and Node y }\end{array}$ & 5 \\
\hline $\begin{array}{l}\text { The difference between the mean of first 50 data points } \\
\text { and the mean of last 50 data points }\end{array}$ & \\
\hline
\end{tabular}

MTI (Moving Target Indication) is utilized on the radar data to remove the background noise and static clutter through a notch filter. For the positive Doppler, the cut-off frequency is $0.0075 \mathrm{~Hz}$, whereas the cut-off frequency of the negative Doppler is $-0.0075 \mathrm{~Hz}$. To map the information to the Doppler-Time domain, STFT (Short Time Fourier Transform) [18]with 0.5s Hamming window and 95\% overlapping between successive FFTs (Fast Fourier Transform) is implemented on the filtered signal. The Doppler spectrogram in Fig. 2 is generated by summing the STFT data among the useful range bins containing contributions from the target. The radar spectrogram includes information on the movement of palm and fingers, which can be helpful to recognize similar gesture transitions such as 0 to 5 or 1 to 5 in our example. Useful radar features are extracted from the Doppler spectrogram according to our previous work on human activities [19]. Two generally important features are in particular the Doppler centroid and bandwidth [20], whose formulae are listed below in (1), (2):

$$
\begin{gathered}
f_{D C}(j)=\frac{\sum_{i} f_{d}(i) S(i, j)}{\sum_{i} S(i, j)} \\
f_{B C}(j)=\sqrt{\frac{\sum_{i}\left(f_{d}(i)-f_{D C}(j)\right)^{2} S(i, j)}{\sum_{i} S(i, j)}}
\end{gathered}
$$

Both centroid and Bandwidth are calculated for every $j^{\text {th }}$ time bin; $f_{d}(i)$ is the Doppler frequency for the $i^{\text {th }}$ Doppler bin, $S(i, j)$ denotes the Doppler spectrogram matrix. The Doppler centroid represents the center of mass of the palm and fingers motions, whereas the bandwidth captures the energy spread surrounding the center of mass; in our case, they are significantly coherent with the fingers trajectory.

To reduce the computational load and increase performances, only the most significant and non-redundant features from radar data are selected using a wrapper method approach, Sequential Feature Selection [21], [22](SFS), in conjunction with a Quadratic-kernel SVM classifier [23].

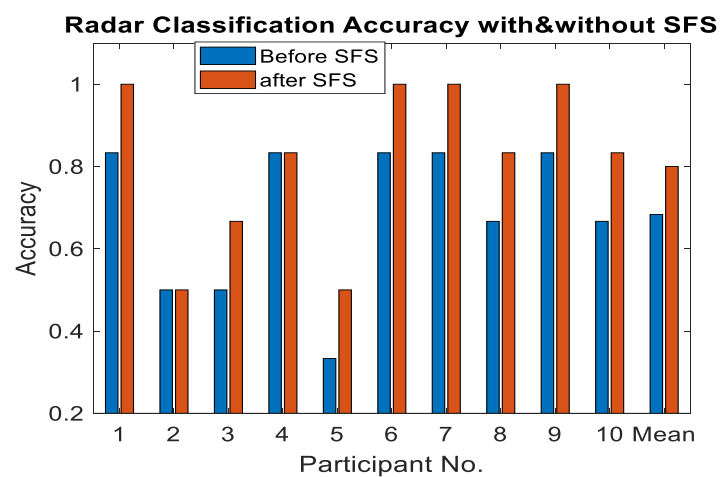

Fig. 3 Radar SFS results with dynamic gesture.

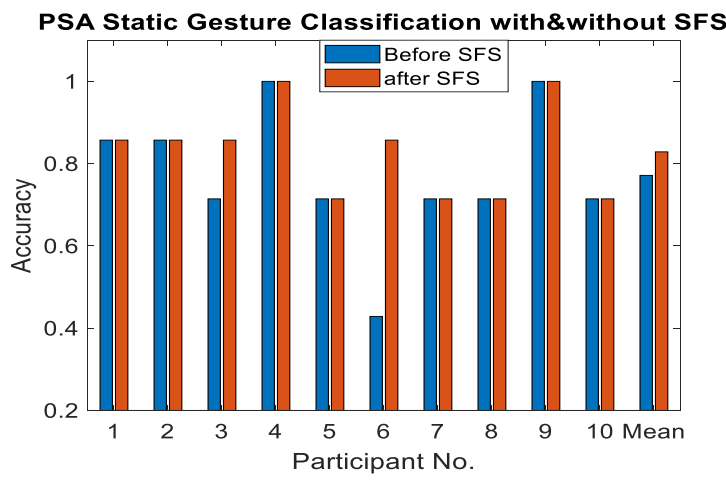

Fig. 4 PSA SFS results with static gesture.

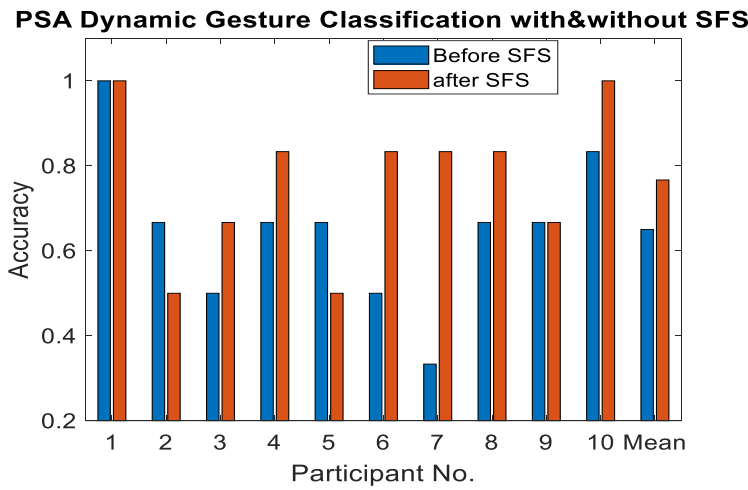

Fig. 5 PSA SFS results with dynamic gesture.

\section{B. General Classification with PSA and Radar}

A Quadratic-kernel SVM is chosen as a robust supervised learning algorithm to train the classification model and identify the testing gestures accordingly. The support vectors construct an optimal hyperplane between the data points distributed in the feature space, whereas a kernel function with the polynomial order equal to 2 is utilized to map the information to a higher dimension. More details of SVM and attached kernel functions can be found at [24], [25].

Different cross-validation methods (e.g. Holdout and Kfold) are utilized in our previous work [19] and literatures [22] to partition the data into training and test set. However, in this paper, 'leaving one participant out' method is introduced for more realism and challenging classification. In this case, one participant is selected for evaluating the classification performance, whereas the data from other participants are utilized for training the SVM classifier 
translating to a 9:1 training to testing ratio. Additionally, the training and test process will continue until every participant is tested upon. The final classification accuracy is the average of all six iterations.

Figs. 6-7 illustrates the correctly classified events (diagonal components) and misclassifications (non-diagonal components) by using 'leaving one participant out' with PSA and radar independently (average across all participants). The columns represent the output class, corresponded to the prediction label, whereas the rows indicate the target class, corresponding to the ground truth.

The sum of the column elements of the confusion matrix is equal to $100 \%$. When we identify the static gestures, it is observed that the main misclassification takes place between ' $\mathrm{G} 1$ ', and 'G2', 'G3 and G4', while few interleaving misclassifications exist between ' $\mathrm{G} 1$ ' and ' $\mathrm{G} 3$ '. The average classification accuracy across all the static gestures (PSA) is approximately $82.9 \%$, whereas for the gesture transitions the results are slightly lower, at about 80\%. The main misclassification in Fig. 6 for the static gestures is between 'G1' and 'G2'; it is due to the similarity of the tendon's pressure when the participant performs ' 0 ' and ' 1 '. In Fig. 7, the main error occurs between ' $0-2$ ' and ' $2-1$ '; apart from that, around $30 \%$ gesture transition ' $1-5$ ' have been misclassified to ' $2-0$ '. This is because each participant has their own individual style to perform the gestures: someone tends to leave their hand flat, which produces a stronger Doppler signature, whereas others tend to create an angle between the hand and the radar line-ofsight, and then the signature is weakened. Therefore, those weaker signatures make the classifier more confuse and cause more misclassifications.

\section{Hierarchical Classification Models (Soft Fusion using Confidence Level)}

Fig. 8 illustrates the first hierarchical model comprised of two classification stages. It is proposed to boost the classification performance of static gestures with the help of radar (radar as the "enhancer" sensor). The first stage of classification takes place after the feature selection, and the classifier will generate a score matrix and the corresponding prediction label. The score matrix contains the confidence level for each individual class and the prediction label is the specific class with the highest confidence level.

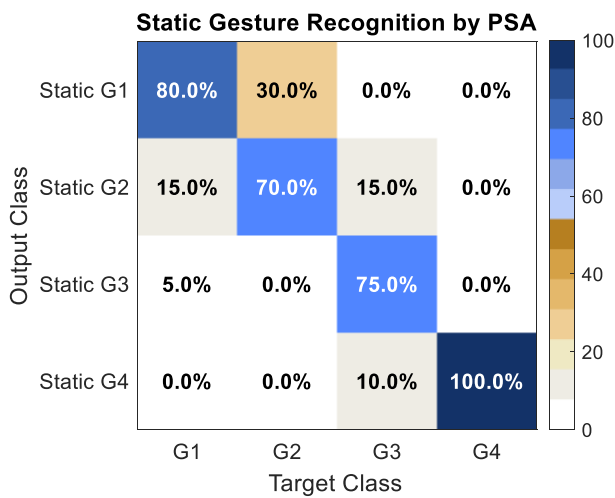

Fig. 6 Confusion matrix of PSA-only for static gesture recognition

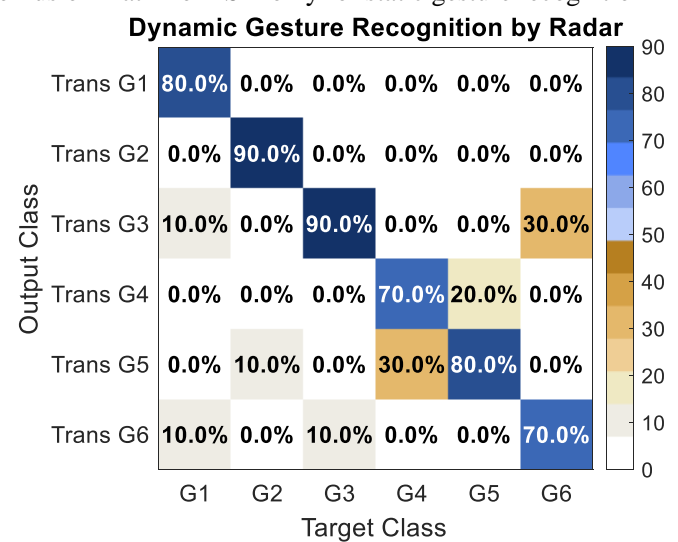

Fig. 7 Confusion matrix of radar-only for gesture transition recognition

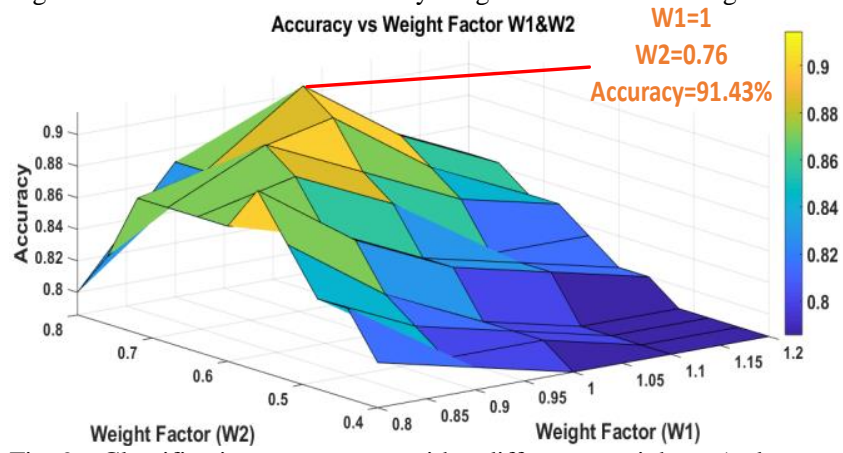

Fig. 9 Classification accuracy with different weights (radar as 'Enhancer').

In this regard, the PSA and radar score matrices have 4 and six columns with respect to the number of classes to recognize, four static gestures for PSA and six transitions for radar.

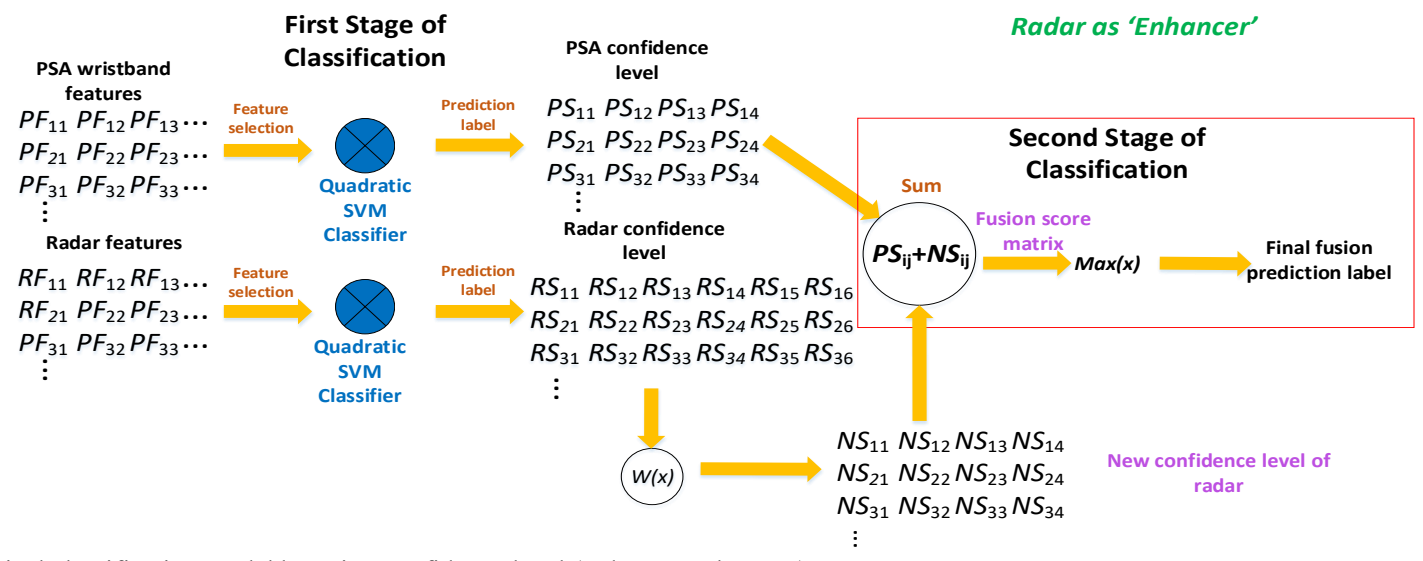

Fig. 8 Hierarchical classification model by using confidence level (radar as 'Enhancer'). 
Given this class imbalance between radar and PSA, the score matrices from different sensors cannot be simply added together. To address this, we assume that in this context the important information is given by the static gesture and not by the transition. Hence, the six-classes (transitions) matrix from the radar can be translated into a four-classes (static gesture) matrix considering the final gesture after each transition. Furthermore, as the radar in this approach acts as an "enhancer" of the PSA to recognise static gestures, a weighted function has been implemented on the new 4-class score matrix to control the radar influence in the In the new confidence level matrix of radar, if the prediction label of the $i^{\text {th }}$ observation is class ' $\mathrm{G} 1$ ', then $N S_{\mathrm{ij}}$ is derived by equation (3):

$$
N S_{i j}=\left\{\begin{array}{l}
W_{1}(j=1) \\
W_{2}-\max \left\{R S_{i 1}, R S_{i 2}, \cdots, R S_{i 6}\right\}(j=2,3,4)
\end{array}\right.
$$

This transform converts a 'six classes' problem to a 'four classes' problem with keeping the original information from radar simultaneously. W1 and W2 denotes two weight factors, used to adjust the impact of radar in the fusion procedure. Increasing W2 or decreasing W1 will strengthen the influence of radar, and vice versa. To determine the best weight factors for the fusion, different numbers of weight factors have been verified and compared in Fig. 9 in terms of classification accuracy. It is reported that the classification accuracy achieves the highest level (approximately 91.43\%) when $\mathrm{W} 1$ and $\mathrm{W} 2$ are set equal to 1 and 0.76 , separately.

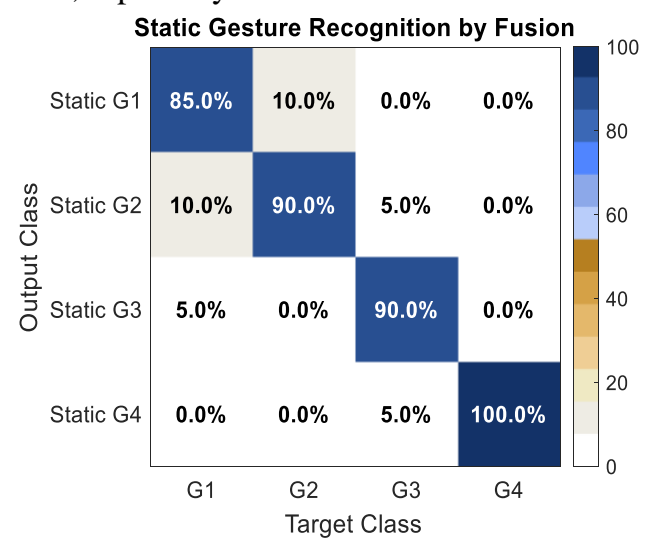

Fig. 10 Confusion matrix of soft fusion (radar as 'Enhancer').

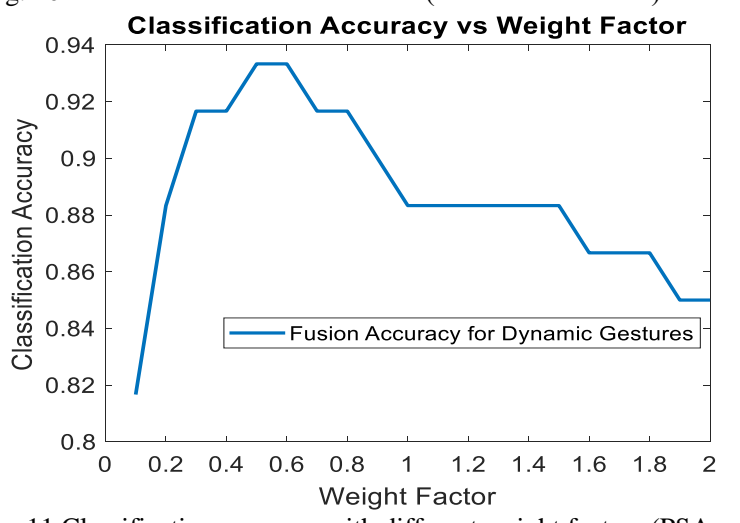

Fig. 11 Classification accuracy with different weight factors (PSA as 'Enhancer')
Fig. 10 shows the classification results (confusion matrix) when the Doppler radar acts as an 'Enhancer' to help the PSA with the static gesture recognition. The mean accuracy across all the participants improves by approximately $8.6 \%$ compared to figure 6 , whereas the misclassification between ' $\mathrm{G} 1$ ' and ' $\mathrm{G} 2$ ' has been reduced to a lower level. The class ' $\mathrm{G} 2$ ' has the worst recognizable rate in the case of using a single sensor as shown in Fig. 6, however, the correctly recognition rate with ' $\mathrm{G} 2$ ' is increased to $90 \%$ (about $20 \%$ improvement) through fusion with Doppler radar.

The PSA sensor could be used as "Enhancer" for the radar to classify transitions between static gestures. In the second classification stage, the confidence level of PSA and radar are weighted and summed to construct the fusion score matrix, whereas the weight function is utilized to control the influence of PSA. The relationship between accuracy and weight between radar and PSA information is shown in Fig. 11 The fusion performance reaches the peak when the ratio of PSA and radar confidence level in the fusion score equals to 0.5 and 0.6.

Fig. 12 illustrates the classification confusion matrix of soft fusion for dynamic gestures. Very high classification performance is obtained for two transitions, whereas some minor misclassifications are still present in the others.

\section{Hierarchical Classification Models (Hard Fusion using Prediction Label)}

Instead of merging the confidence level of different sensors in the second stage of hierarchical model, the hard fusion of radar and PSA takes place between the prediction labels through a probability combiner. There are several potential combiners in the literature [14], [26], including the majority voting system or weighted voting system used in our previous work [19], Recall combiner and Naïve Bayes combiner [14]. The voting-based system is not suitable due to the decision clashes in our case scenario, whereas it is not ideal to use Recall combiner in binary classes problems [26] since the performance of Recall combiner is proportional to the number of classifiers (here we only have two classifiers from two sensors). Hence, in this paper, we chose Naïve Bayes are unavoidable combiner to calculate the posterior probability of each class through the prediction label and confusion matrix of the individual sensor. In this case, the probability of certain class after fusion [14] is obtained by the equation (4) below:

$$
\log P\left(C_{k} \mid d\right) \propto \log \left(P\left(C_{k}\right)\right)+\sum_{m=1}^{N} \log \left(p_{m, C_{m}, k}\right)
$$

Where $P\left(C_{k} \mid d\right)$ is the probability we are interested in, denoted for the possibility that class $C_{k}$ is the true class.

$P\left(C_{k}\right)$ represents the number of classifiers which suggested $C_{k}$ as the prediction label. The classifier used belongs to a classifier ensemble whose length is equal to $N$. $p_{m, C_{m}, k}$ refers to the confusion matrix element corresponding to classifier $m$, row $C_{m}$ and column $k$. The 
final prediction label is the class with the highest posterior probability. Compared with soft fusion, hard fusion is more efficient computationally and saves time for selecting the optimal weight function.

For the gesture transitions, the hard fusion yields approximately $95 \%$ classification accuracy, whereas the static gesture recognition through hard fusion indicates no significant improvement. Fig. 13 reports the hard fusion

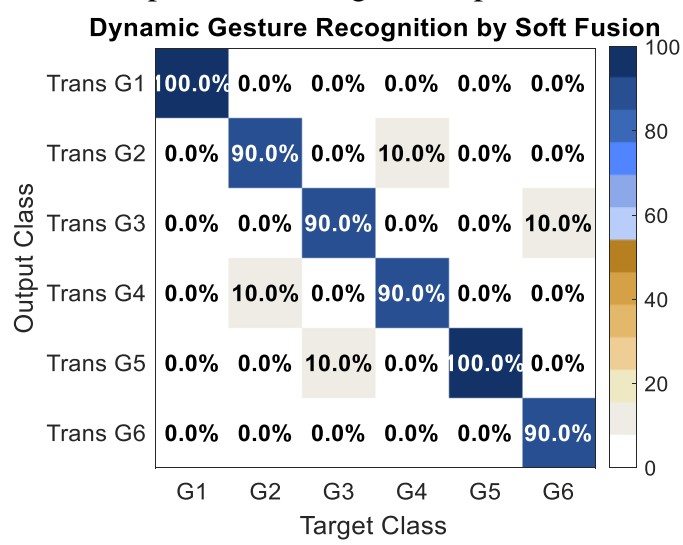

Fig. 12 Confusion matrix of soft fusion (PSA as 'Enhancer').

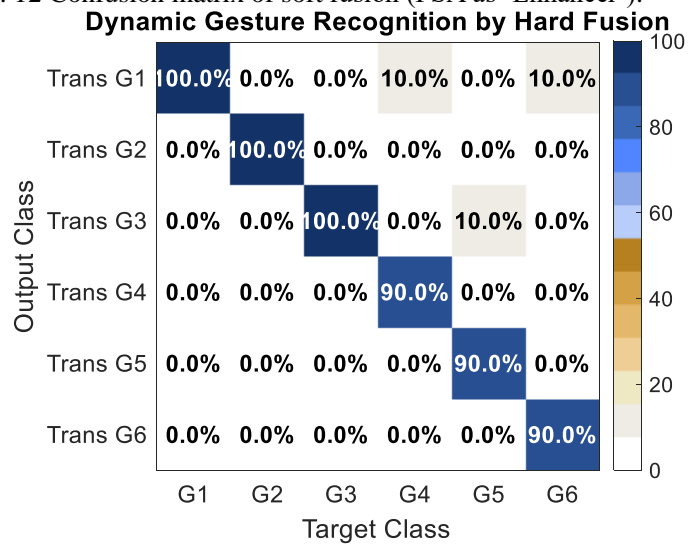

Fig. 13 Classification results of gesture transition recognition using hard fusion.
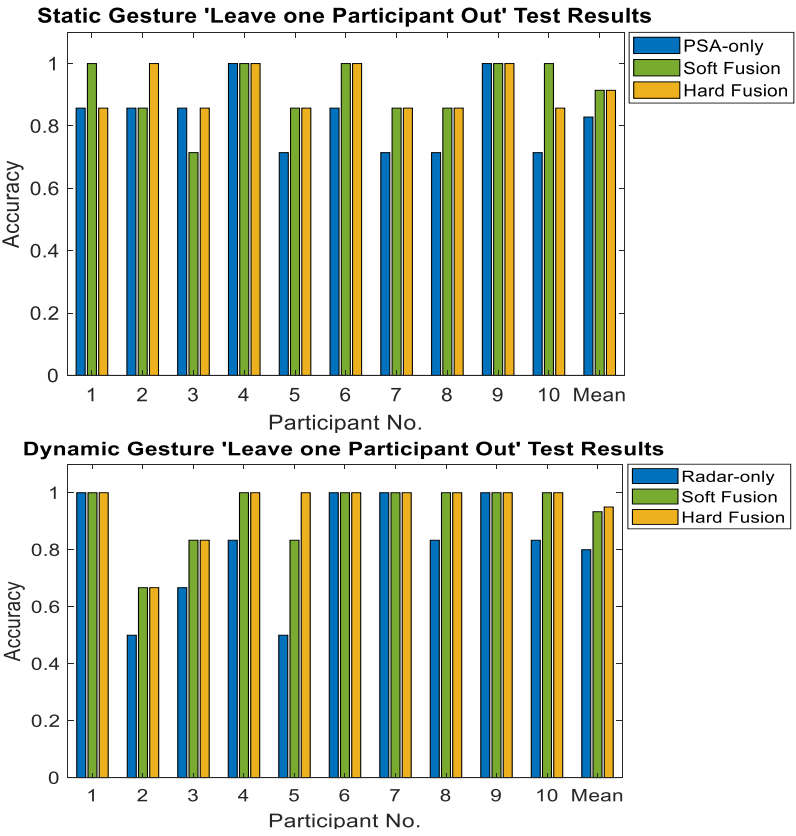

Fig. 14 'Leave one participant out' test results (top: static gesture, bottom: dynamic gesture). results of gesture transitions using a Naïve Bayes combiner. Compared to the soft fusion results, the accuracy of hard fusion approach increased by about $1.7 \%$, where less misclassifications exist between Transitions ' $0-5$ ' and ' $0-2$ ', '2-0' and ' $2-1$ ', ' $0-5$ ' and ' $1-5$ '. Those misclassified gesture transitions are similar and not easy to resolve, even combining the perspectives of both sensors.

Fig. 14 summarises the results for static and dynamic gesture recognition using a single sensor, and the soft fusion and hard fusion approaches with the hierarchical models proposed. The classification accuracy increases about $8.6 \%$ and $13.3 \%$ for static and dynamic gestures through soft fusion, while hard fusion significantly reduces the variance across all the participants as in Fig. 15; this also provides a subsequent improvement of $1.7 \%$ in the dynamic gesture recognition. In other words, an advantage of hard fusion is the enhancement in the stability of the hierarchical classification model across the different participants.

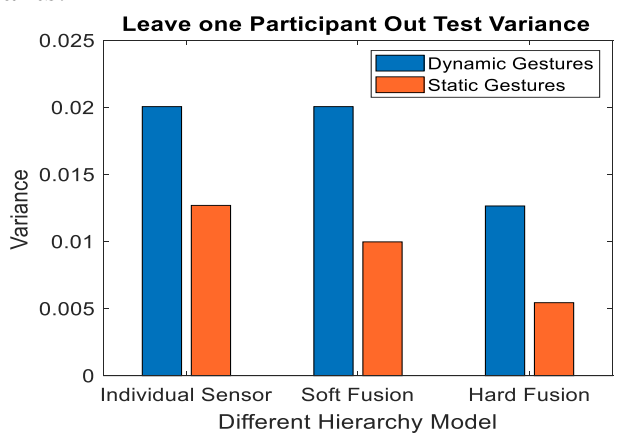

Fig. 15 Accuracy variance of different hierarchy model.

\section{CONCLUSION}

This paper proposed a novel hierarchical sensor fusion architecture using outputs from the pressure sensor and Doppler radar. In this hierarchy of sensors, one can act as "enhancer" of the other depending on the specific application, for example whether it is more relevant capturing static gestures (radar enhancing the PSA) or dynamic gestures (PSA enhancing radar). Two fusion schemes based on soft and hard fusion are also implemented in the hierarchical model, and the results show significant improvement compared to the case of single sensor used individually.

Future work can include the generalisation of these approaches to cases with more sensors and greater variability in terms of the number of participants and gestures performed. As the classification algorithm was relatively simple in this case, more elaborated approaches based on deep learning can be explored. These include Convolutional Neural Network (CNN) [27] for image processing and Recurrent Neural Network (RNN) for timedependent correlations, or combinations of the two. Without resorting to deep learning, extending the number of simpler classifiers could increase performances through other possible fusion/ensemble methods (e.g. voting system and Recall combiner). Finally, the implementation of the algorithm in a real-time setting leveraging on FPGA platforms [28]or compact high-performance computing boards can also be very interesting. 


\section{ACKNOWLEDGMENT}

The authors are grateful to the volunteers who helped with the data collection and to D. Ciuonzo at the University of Naples for discussions and ideas on data fusion techniques.

\section{REFERENCES}

[1] G. Li, R. Zhang, M. Ritchie, and H. Griffiths, "Sparsity-based dynamic hand gesture recognition using micro-Doppler signatures," in Radar Conference (RadarConf), 2017 IEEE, pp. 928-931, 2017.

[2] D. Wu, L. Pigou, P. Kindermans, N. D. Le, L. Shao, J. Dambre, and J. Odobez, "Deep dynamic neural networks for multimodal gesture segmentation and recognition," IEEE Trans. Pattern Anal. Mach. Intell., vol. 38, no. 8, pp. 1583-1597, 2016.

[3] X. Liang, R. Ghannam, and H. Heidari, "Wrist-worn gesture sensing with wearable intelligence," IEEE Sens. J., vol. 19, no. 3, pp. 10821090,2018

[4] P. Molchanov, S. Gupta, K. Kim, and K. Pulli, "Multi-sensor system for driver's hand-gesture recognition," in Automatic Face and Gesture Recognition (FG), 2015 11th IEEE International Conference and Workshops on, vol. 1, pp. 1-8, 2015.

[5] R. Wen, W.-L. Tay, B. P. Nguyen, C.-B. Chng, and C.-K. Chui, "Hand gesture guided robot-assisted surgery based on a direct augmented reality interface," Comput. Methods Programs Biomed., vol. 116, no. 2, pp. 68-80, 2014.

[6] C. Zhu and W. Sheng, "Wearable sensor-based hand gesture and daily activity recognition for robot-assisted living," IEEE Trans. Syst. Man, Cybern. A Syst. Humans, vol. 41, no. 3, pp. 569-573, 2011.

[7] H. Heidari, E. Bonizzoni, U. Gatti, F. Maloberti, and R. Dahiya, "CMOS vertical Hall magnetic sensors on flexible substrate," IEEE Sens. J., vol. 16, no. 24, pp. 8736-8743, 2016.

[8] P. Jung, G. Lim, S. Kim, and K. Kong, "A wearable gesture recognition device for detecting muscular activities based on airpressure sensors," IEEE Trans. Ind. Informatics, vol. 11, no. 2, pp. 485-494, 2015.

[9] G. Jeong, P. H. Truong, and S. Choi, "Classification of three types of walking activities regarding stairs using plantar pressure sensors," IEEE Sens. J., vol. 17, no. 9, pp. 2638-2639, 2017.

[10]H. Wang, D. Zhang, Y. Wang, J. Ma, Y. Wang, and S. Li, "RT-Fall: A real-time and contactless fall detection system with commodity WiFi devices," IEEE Trans. Mob. Comput., vol. 16, no. 2, pp. 511-526, 2017.

[11]E. Cippitelli, F. Fioranelli, E. Gambi, and S. Spinsante, "Radar and RGB-depth sensors for fall detection: a review," IEEE Sens. J., vol. 17, no. 12, pp. 3585-3604, 2017.

[12]H. Li, A. Shrestha, F. Fioranelli, J. L. Kernec, and H. Heidari, "Hierarchical classification on multimodal sensing for human activity recogintion and fall detection," in 2018 IEEE SENSORS proceedings, pp. 1-4, 2018.

[13]C. Chen, R. Jafari, and N. Kehtarnavaz, "A real-time human action recognition system using depth and inertial sensor fusion," IEEE Sens. $J$, vol. 16, no. 3, pp. 773-781, 2016.

[14]L. Kuncheva and J. Rodríguez, A weighted voting framework for classifiers ensembles, vol. 38. 2014.

[15] V. C. Chen, W. J. Miceli, and D. Tahmoush, Radar micro-Doppler signatures: processing and applications. The Institution of Engineering and Technology, 2014.

[16]H. S. AlZubi, S. Gerrard-Longworth, W. Al-Nuaimy, Y. Goulermas, and S. Preece, "Human activity classification using a single accelerometer," in Computational Intelligence (UKCI), 2014 14th UK Workshop on, pp. 1-6, 2014.

[17]H. Li, A. Shrestha, H. Heidari, J. L. Kernec, and F. Fioranelli, "Magnetic and Radar Sensing for Multimodal Remote Health Monitoring," IEEE Sens. J., p. 1, 2018.

[18]D. E. Barrick, "FM/CW radar signals and digital processing," National Oceanic and Atmospheric Administration Boulder co Wave Propagation Lab, 1973.

[19]H. Li, A. Shrestha, H. Heidari, J. L. Kernec, and F. Fioranelli, "A multisensory approach for remote health monitoring of older people," IEEE J. Electromagn. RF Microwaves Med. Biol., vol. 2, no. 2, pp. $102-108,2018$.
[20]F. Fioranelli, M. Ritchie, and H. Griffiths, "Centroid features for classification of armed/unarmed multiple personnel using multistatic human micro-Doppler," IET Radar, Sonar Navig., vol. 10, no. 9, pp. $1702-1710,2016$

[21] A. Jain and D. Zongker, "Feature selection: Evaluation, application, and small sample performance," IEEE Trans. Pattern Anal. Mach. Intell., vol. 19, no. 2, pp. 153-158, 1997.

[22]K. Altun and B. Barshan, "Human activity recognition using inertial/magnetic sensor units," in International Workshop on Human Behavior Understanding, pp. 38-51, 2010.

[23]G. Roffo, "Feature selection library (MATLAB toolbox)," arXiv Prepr. arXiv1607.01327, 2016.

[24] T. D. Bufler and R. M. Narayanan, "Radar classification of indoor targets using support vector machines," IET Radar, Sonar Navig., vol. 10, no. 8, pp. 1468-1476, 2016.

[25]C. Cortes and V. Vapnik, "Support-vector networks," Mach. Learn., vol. 20 , no. 3 , pp. $273-297,1995$.

[26]G. Aceto, D. Ciuonzo, A. Montieri, and A. Pescapé, "Multiclassification approaches for classifying mobile app traffic," J. Netw. Comput. Appl., vol. 103, pp. 131-145, 2018.

[27]M. Zeng, L. T. Nguyen, B. Yu, O. J. Mengshoel, J. Zhu, P. Wu, and J. Zhang, "Convolutional Neural Networks for human activity recognition using mobile sensors," in 6th International Conference on Mobile Computing, Applications and Services, pp. 197-205, 2014.

[28] S. Rajko, G. Qian, T. Ingalls, and J. James, "Real-time gesture recognition with minimal training requirements and on-line learning," in 2007 IEEE Conference on Computer Vision and Pattern Recognition, pp. 1-8, 2007.

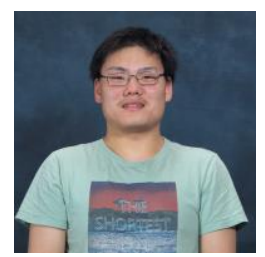

H. $\mathbf{L i}$ is currently a $2^{\text {nd }}$ year $\mathrm{PhD}$ student at the School of Engineering, University of Glasgow. He received his BEng in Electrical \& Electronic Engineering from Northumbria University at Newcastle and MS in Communication \& Signal Processing from University of Newcastle, in 2015 and 2016 respectively. $\mathrm{He}$ is working on information fusion of multiple sensing technologies for assisted living applications and gesture recognition.

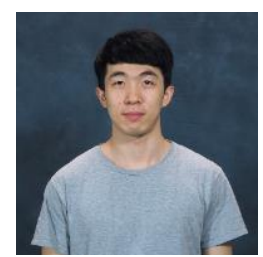

X. Liang is a PhD Student in Microelectronics $\mathrm{Lab}$ (meLAB) at Electronic and Electrical Engineering with research interests in the field of neuromorphic chip. Following his MSc (with distinction) from University of Glasgow in 2017, He has spent a year in the Zengyi Technology \& National Instrument, Beijing as placement. He received his BEng from Guangzhou College of South China University of Technology, China. He is an IEEE student member.

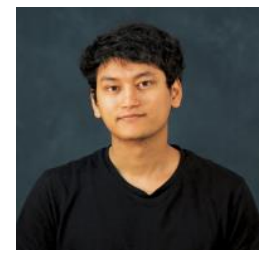

A. Shrestha is currently a PhD student at the School of Engineering, University of Glasgow, working on application of radar systems and radar signal processing for assisted living with machine learning.

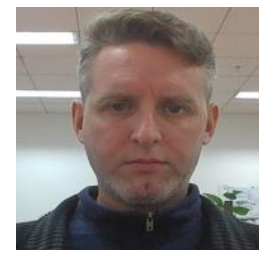

J. Le Kernec (SM'17) received the B.Eng./M.Eng. degrees in electronic engineering from Cork Institute of Technology, Ireland, in 2004 and 2006, and the $\mathrm{PhD}$ degree in electronic engineering from University Pierre and Marie Curie, France, in 2011. He is currently a Lecturer with the School of Engineering, University of Glasgow. He is also seconded as a Lecturer in 
the University of Electronic Science and Technology of China and an adjunct associate professor in University Cergy-Pontoise in France in the ETIS lab. His research interests include radar system design, software defined radio/radar, signal processing, and health applications. orcid.org/0000-0003-2124-6803

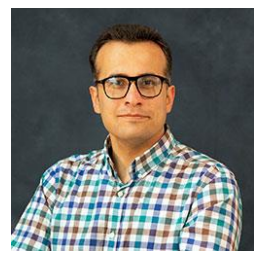

Hadi Heidari (M'15-SM'17) is a Lecturer (Assistant Professor) and lead the Microelectronics Lab (meLAB) in the School of Engineering at the University of Glasgow, UK. He has authored over 100 publications in peer reviewed journals (e.g. IEEE Solid-State Circuits Journal, Trans. Circuits and Systems I and IEEE Trans. Electron Devices) and in international conferences. He has been the recipient of a number of awards including the IEEE CASS Scholarship (NGCAS'17 conference), Silk Road Award from the Solid-State Circuits Conference (ISSCC'16), Best Paper Award from the IEEE ISCAS'14 conference, Gold Leaf Award from the IEEE PRIME'14 Conference and Rewards for Excellence prize from the University of Glasgow (2018). He involves in the organising committees of the IEEE PRIME'15,'19, SENSORS'16,'17, IEEE NGCAS'17, BioCAS'18, ISCAS'20,'23 conferences and chairing three special sessions at ISCAS'16,'17,'18,'19. He is an IEEE Senior Member, an Editor for the Elsevier Microelectronics Journal, an Associate Editor for the Elsevier Microelectronics Journal, Associate Editor of IEEE Journal of Electromagnetics, RF and Microwaves in Medicine and Biology and IEEE Access, and lead Guest Editor for four journal special issues. He is member of the IEEE Circuits and Systems Society Board of Governors (2018-21), and a Member-at-Large at the IEEE Sensors Council (2020-21). $\mathrm{He}$ is the General Chair of the IEEE International Conference on Electronics Circuits and Systems (ICECS) 2020.

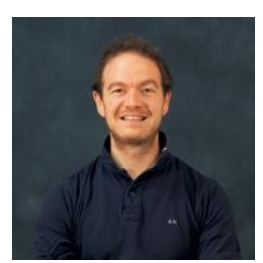

F. Fioranelli (M'15-SM'19) is currently a Lecturer (Assistant Professor) at the School of Engineering, University of Glasgow. $\mathrm{He}$ received his $\mathrm{PhD}$ at Durham University, UK, in 2014, and was a postdoctoral Research Associate at University College London in 2014-2016, prior to joining the University of Glasgow. His research interests include development of radar systems and radar signal processing for applications including human signatures analysis for healthcare and security, drones and UAVs detection and classification, automotive radar, wind farm and sea clutter characterization. Dr Fioranelli is a member of the IEEE, a Chartered Engineer (CEng) through the IET, and regularly acts as reviewers for academic journals in the domain of radar sensing, such as IEEE TAES, IEEE Sensors, IEEE TGRS, and IET RSN. 